\title{
A contratualização nos Hospitais de Ensino no Sistema Único de Saúde brasileiro
}

\author{
The contracting of teaching hospitals \\ in the Brazilian Unified Health System
}

Sheyla Maria Lemos Lima ${ }^{1}$

Francisco Javier Uribe Rivera ${ }^{1}$

${ }^{1}$ Departamento de Administração e Planejamento em Saúde, Escola Nacional de Saúde Pública, Fundação Oswaldo Cruz, Ministério da Saúde. Rua Leopoldo Bulhões 1480/717, Manguinhos.

21041-210 Rio de Janeiro RJ.slemos@ensp.fiocruz.br
Abstract This study identifies the potential and limitations of contracting to improve health care management, accountability and quality, and expand the participation of teaching hospitals in the health service network in the context of the Restructuring Program of Teaching Hospitals in the Brazilian Unified Health System. It is a case study of four teaching hospitals and their contracting State Health Departments. According to the hospital managers, the association is weak between contracting and the presence of mechanisms for hospital insertion into the health service network with practices and structures for managerial and healthcare qualification in the hospital. More structured hospitals in managerial and healthcare terms were more structured between contracting and the State Health Department. There was an increase in production of medium complexity outpatient care and a decrease in primary healthcare procedures. The proposal is for ongoing managerial development of the hospital and of the State Health Department, review of the operational plan, budgeting, monitoring mechanisms and an incentive system, bonding in the teams, among others.

Key words Contracting, Teaching hospitals, Accountability, Managerial improvement, Healthcare improvement, Agency theory
Resumo São discutidas as possibilidades e os limites da contratualização para a melhoria do desempenho, o incremento da prestação de contas, o aprimoramento da gestão, a melhoria da assistência e a maior inserção dos hospitais de ensino na rede de serviços no âmbito do Programa de Reestruturação dos Hospitais de Ensino no Sistema Único de Saúde/SUS. Quatro hospitais contratados e suas secretarias contratantes são entrevistados. Segundo os dirigentes de hospitais, é frágil a associação entre contratualização e a presença de mecanismos de inserção na rede, de práticas e estruturas de qualificação assistencial e gerencial nos hospitais. Hospitais mais estruturados assistencial e gerencialmente apresentaram uma contratualização mais estruturada com a secretaria. Houve um aumento de produção da média complexidade ambulatorial e uma diminuição dos procedimentos de atenção básica em todos os hospitais. Sugere-se o desenvolvimento gerencial contínuo do hospital e da secretaria, a revisão do plano operativo, orçamento, mecanismos de monitoramento e sistema de incentivos, pactuação com as equipes, dentre outros.

Palavras-chave Contratualização, Hospitais de ensino, Prestação de contas, Melhoria gerencial, Melhoria assistencial, Teoria da Agência 


\section{Introdução}

Os arranjos contratuais têm sido cada vez mais utilizados, em vários países, entre autoridades governamentais de saúde e prestadores, privados ou estatais, de atenção básica e hospitalar com o intuito de melhorar o desempenho dos prestadores e incrementar a prestação de contas de resultados para usuários, financiadores e governos. A suposição subjacente é que os resultados insatisfatórios, em parte, são determinados pela insuficiente prestação de contas e pelo repasse de recursos financeiros não condicionado ao desempenho ${ }^{1-7}$.

Os arranjos contratuais necessariamente envolvem duas partes - o ente contratante/financiador e o(s) ente(s) contratado(s)/prestador(es). Constituem-se em mecanismos de coordenação nos sistemas de saúde públicos que separaram as funções de financiamento/compra e de regulação, da função de prestação de serviços e naqueles em que a referida separação não ocorreu.

Conforma-se no tripé de sustentação dos arranjos contratuais, um plano de atividades com estimativa de recursos para sua execução, os mecanismos de acompanhamento e avaliação e um sistema de incentivos, que condicione o repasse de recursos ao alcance de resultados pré-definidos e acordados.

No Brasil, os arranjos contratuais estão no cerne das discussões sobre alternativas para a administração pública tendo em vista a melhoria do desempenho e da prestação de contas dos prestadores de serviços de saúde. Estão sendo propostos e/ou utilizados como instrumentos de coordenação e ligação do núcleo central da administração pública, com seus próprios entes internos já existentes e/ou com novas modalidades jurídico-administrativas, público ou privadas, prestadores de serviços de saúde. Quaisquer alternativas - fundações estatais de direito privado, organizações sociais, ou até mesmo a recente proposição, ainda não aprovada no Senado, a Empresa Brasileira de Serviços Hospitala$\mathrm{res}^{8}$, destinada exclusivamente à gestão dos hospitais de ensino federais -, o contrato é o instrumento que vincula a administração direta, ente financiador, ao ente prestador.

Mais recentemente ainda, o governo federal e o MS ${ }^{9}$, regulamentando o Sistema Único de Saúde/SUS, propuseram o estabelecimento do "Contrato Organizativo de Ação Pública da Saúde" entre o Ministério da Saúde (MS) e os entes federativos, articulados em Regiões de Saúde, com a finalidade de constituir redes de atenção à saúde, por meio da integração dos planos de saúde dos entes em contrato, definindo metas, indicadores e critérios de avaliação de desempenho associados ao repasse de recursos financeiros.

No Brasil, desde o final de 1990, os arranjos contratuais entre hospitais e unidades de atenção básica com secretarias de saúde estaduais ou municipais vêm sendo estabelecidos. As experiências mais consolidadas no âmbito hospitalar são as das secretarias de saúde dos estados de Minas Gerais e São Paulo e do MS com os Programas de Reestruturação dos Hospitais de Ensino ${ }^{10,11}$, Hospitais Filantrópicos ${ }^{12}$ e Hospitais de Pequeno Porte ${ }^{13}$. Apesar do reconhecimento dessas experiências e das novas iniciativas existentes, este artigo exclusivamente analisará a política de contratualização dos Hospitais de Ensino do MS.

Em 2004 e 2005, o MS e o Ministério da Educação implementaram uma política de reestruturação para os hospitais de ensino, induzindo o estabelecimento da contratualização entre eles e as secretarias de saúde estaduais e/ou municipais gestoras da rede de serviços ${ }^{10,11}$.

O Programa de Reestruturação dos Hospitais de Ensino envolvendo os hospitais federais ${ }^{10}$ e os demais hospitais de ensino de natureza pública ou privada ${ }^{11}$ tem como objetivos a qualificação e o desenvolvimento da assistência, da gestão, do ensino, da pesquisa e da avaliação tecnológica em saúde/ATS, tendo em vista o SUS.

No campo assistencial preconiza o aumento de procedimentos de média e alta complexidade e a diminuição dos de atenção básica, a introdução de modalidades assistenciais que diminuam o tempo de internação, a oferta de 100\% dos leitos para o SUS, a inserção do hospital no sistema de urgência e emergência, a introdução de práticas e estruturas qualificadoras da assistência, dentre outras diretrizes. No campo gerencial, recomenda a introdução de estruturas colegiadas, de planejamento participativo, dentre outras ações. Há também diretrizes para o ensino, pesquisa e ATS as quais foram excluídas do escopo do artigo.

São condições obrigatórias para ingresso no Programa, a certificação e a contratualização. A certificação, competência conjunta do MEC e do MS, renovada periodicamente, estabelece critérios claros e precisos, até então inexistentes, nos campos da assistência, gestão, ensino e pesquisa para o hospital ser considerado de ensino ${ }^{14}$.

A contratualização entre secretaria municipal e/ou estadual e o hospital institui um processo inovador de negociação sobre as atividades a serem desenvolvidas pelo hospital, expressas em 
um Plano Operativo Anual (POA), os mecanismos de monitoramento e avaliação, os recursos envolvidos e os critérios de repasse, incluindo ainda outras exigências ${ }^{10}$.

Os convênios/contratos têm duração máxima de cinco anos, com renovação anual do POA e orçamento, os quais podem ser revistos a qualquer momento através de Termo Aditivo, desde que acordado entre as partes ${ }^{10}$.

O POA discrimina todos os serviços ofertados, a estrutura tecnológica e a capacidade instalada do hospital. Nele, constam as metas e indicadores de produção (internações, consultas ambulatoriais, atendimentos de urgência e emergência e dos serviços de apoio ao diagnóstico e tratamento); de inserção na rede; de qualificação da assistência; de aperfeiçoamento da gestão; de atuação no ensino, na formação e na educação permanente dos profissionais do hospital e da rede; e de desenvolvimento de pesquisa e de avaliação tecnológica em saúde ${ }^{10}$.

O POA e o convênio/contrato devem ser monitorados e avaliados, ao menos uma vez ao mês, por uma Comissão de Acompanhamento de Convênios/Contratos, constituída e coordenada pela Secretaria de Saúde, com representação do hospital, da comunidade acadêmica e dos usuários ${ }^{10}$.

O ingresso do hospital no Programa é estimulado pelo recebimento de um recurso extraorçamentário, o incentivo à contratualização (IAC). Além disso, os hospitais passam a ter um orçamento misto, composto por um valor global, fixo e conhecido antecipadamente, relativo aos procedimentos de média complexidade, e por um valor variável, referente aos procedimentos de alta complexidade e estratégicos, repassado retrospectivamente segundo o número de procedimentos realizados. O valor pré-definido é subdividido em um componente fixo e outro variável diretamente vinculado ao desempenho ${ }^{15}$.

Os componentes, fixo e variável, do valor global devem corresponder respectivamente a $85 \%$ e a $15 \%$. O repasse da parte fixa deve ser realizado através de pagamentos mensais, de forma independente de qualquer avaliação e o da parte variável deve depender de avaliação baseada em trinta indicadores, cada um com parâmetro estabelecido e pontuação definida, totalizando 32 pontos ${ }^{15}$. É desejado que o componente variável seja gradativamente aumentado até $50 \%$ dos recursos do valor global ${ }^{15}$.

A experiência é relativamente recente e, até o momento, poucos estudos evidenciam os resultados advindos com a introdução da contratua- lização nos hospitais de ensino. É nessa perspectiva que esse artigo pretende contribuir, considerando a contratualização em quatro hospitais de ensino do Programa de Reestruturação dos Hospitais de Ensino no Sistema Único de Saúde/SUS, ao identificar a relação da contratualização com a melhoria do desempenho, o incremento da prestação de contas, o aprimoramento da gestão, a melhoria da assistência e a maior inserção dos hospitais de ensino na rede de serviços de saúde, diretrizes do referido Programa. Além disso, caracterizar o processo de contratualização considerando as recomendações do Programa; e, por fim, identificar os aspectos facilitadores, as dificuldades, os benefícios e as proposições de ajustes à contratualização segundo a percepção dos dirigentes das secretarias contratantes e dos hospitais contratados.

\section{Métodos}

Caracteriza-se como um estudo exploratório de quatro casos de contratualização, entre a secretaria de saúde contratante e hospital de ensino contratado. Utilizaram-se dois critérios de escolha dos casos: o grau de estruturação da contratualização a partir da percepção da Coordenação-Geral da Atenção Hospitalar do Departamento de Atenção Especializada da Secretaria de Atenção a Saúde do MS (DAE/SAS/MS), responsável pela implementação do Programa, e o tempo desde o estabelecimento da primeira contratualização, até o momento de realização do estudo.

Foram utilizados os dados do cadastro dos hospitais de ensino contratualizados do MS, atualizado em 12/01/2009, do Cadastro Nacional de Estabelecimentos de Saúde (CNES) do MS, atualizado em abril de 2009 para caracterizar porte e complexidade dos hospitais, e do Sistema de Informações Hospitalares (SIH) e Ambulatoriais (SIA) do MS, relativos a 2004 e 2007, para caracterizar a alteração de produção e do tipo de procedimento, além de alguns indicadores hospitalares.

Foram entrevistados 1 dirigente da Coordenação Hospitalar no MS, 5 dirigentes de hospitais de ensino (diretores gerais e/ou assessores de direção) e 5 gestores de secretarias estaduais e municipais de saúde (assessores específicos para os hospitais de ensino, de planejamento e dirigentes da regulação), todos integrantes de comissões de acompanhamento da contratualização.

Com os dirigentes hospitalares utilizou-se um questionário com questões fechadas para identificar a relação da contratualização com práticas 
e estruturas assistenciais e gerenciais existentes extraídas do Programa do MS e de um sistema de classificação do desenvolvimento gerencial aplicado em estudo sobre os hospitais filantrópicos no Brasil. Desse sistema foram consideradas algumas estruturas de direção e planejamento, de gestão de recursos humanos, de logística e de tecnologia de informações ${ }^{16}$. Além disso, foi caracterizada a contratualização a partir das diretrizes do Programa.

Todos os entrevistados identificaram aspectos facilitadores, dificuldades, benefícios e ajustes para o aprimoramento da contratualização através de questões abertas.

As respostas às questões fechadas estão descritas indicando a presença ou a ausência da estrutura e/ou prática e se foram implantados em função da contratualização.

As respostas às questões abertas foram agrupadas segundo a similaridade de conteúdo e são apresentadas associadas, quando possível, à bibliografia internacional que analisa os problemas e os avanços obtidos, até então, com as experiências de contratualização.

Não foram abordadas outras dimensões contidas no Programa (ensino, pesquisa e avaliação tecnológica em saúde) e não foram considerados os contratos e respectivos Planos Operativos Anuais (POA) dos hospitais.

O hospital contratado e a secretaria contratante são identificados por siglas com um mesmo número.

Os resultados apresentados se referem à percepção dos entrevistados, exceto àqueles relativos aos dados dos sistemas oficiais de informação do MS.

As entrevistas foram realizadas de março a outubro de 2008 e uma delas foi complementada em março de 2009. Todos os dirigentes foram entrevistados pela mesma pesquisadora e todos, exceto um, revisaram as informações prestadas.

Esse projeto foi aprovado pelo CEP/ENSP.

\section{Resultados}

\section{Caracterização assistencial e gerencial dos hospitais de ensino e sua relação com a contratualização}

Com base no CNES, os quatro hospitais são gerais, de grande porte, ofertando mais de $90 \%$ dos leitos totais ao SUS, a exceção do HE2 que no caso específico dos leitos intensivos ofertava $50 \%$ para o SUS. Os hospitais possuem complexida- des distintas quanto à oferta de cuidados intensivos, aos tipos de transplantes realizados e à oferta de procedimentos estratégicos e de alta complexidade ambulatorial. O HE2 é o mais complexo - único que possui leitos intensivos Tipo III (classificação do MS segundo adensamento tecnológico), realiza maior diversidade de transplantes e oferta todos os tipos de procedimentos estratégicos e de alta complexidade ambulatorial. O HE3 é menos complexo - só possui leitos intensivos Tipo I, realiza transplante de córnea e rins e não realiza radioterapia e nem ressonância magnética. O HE1 e HE4 parecem ter complexidades similares e mais próximas ao HE2.

Segundo os dados do Sistema de Informações Hospitalares do MS (SIH/MS), de 2004 para 2007, todos os hospitais diminuíram os procedimentos de atenção básica e incrementaram os de média complexidade ambulatorial. A contratualização parece ter estimulado a produção ou ao menos a um melhor registro, e também ter alterado o tipo de procedimento conforme preconizado pelo Programa de Reestruturação. Apesar disso, somente os dirigentes do HE1 e do HE3 associaram a alteração de produção à contratualização (Tabela 1).

Considerando o mesmo período, os hospitais diminuíram o tempo médio de internação, exceto no HE2; a taxa de mortalidade também decresceu, exceto no HE3 (SIH/MS). Um dirigente não atribuiu as alterações à contratualização e os demais não souberam informar.

A contratualização não alterou a disponibilização de leitos, exames e consultas para a secretaria, e também não influenciou a inserção dos serviços de urgência/emergência na rede, o que era desejado pelo Programa.

Quanto às modalidades que concorrem para diminuir o tempo de internação, nenhum dirigente de hospital associou a implantação desses serviços à contratualização. A cirurgia ambulatorial é a única modalidade informada pelos quatro hospitais; o hospital dia foi referido pelo HE2, HE3 e HE4; e a atenção domiciliar pelo HE2 e HE4.

No que se refere às estruturas e práticas qualificadoras da assistência, os quatro hospitais informaram a presença da maior parte delas (mais de $70 \%$ dos 35 itens) sendo que quase todas foram implantadas independentemente da contratualização (Tabelas 2a e 2b).

Quanto às estruturas e práticas qualificadoras da gestão, observa-se que o HE1 e o HE2 estão bem mais estruturados (mais de $80 \%$ dos 17 itens), do que o HE3 e o HE4 (menos de 60\%) (Tabela 3). 
Tabela 1. Produção por grupos de procedimentos em 2004 e 2007 nos Hospitais de Ensino - $2008^{*}$.

\begin{tabular}{|c|c|c|c|c|c|c|}
\hline \multirow[b]{2}{*}{ Grupos de procedimentos } & \multicolumn{3}{|c|}{ HE1 } & \multicolumn{3}{|c|}{ HE2 } \\
\hline & 2004 & 2007 & Variação & 2004 & 2007 & Variação \\
\hline \multicolumn{7}{|l|}{ Internação } \\
\hline Alta complexidade internação & 2.471 & 2.041 & $-17,4 \%$ & 5.249 & 7.306 & $+39.2 \%$ \\
\hline Internação estratégica & 604 & 656 & $+8,6 \%$ & 2.087 & 2.315 & $+10,9 \%$ \\
\hline Média complexidade internação & 17.115 & 16.631 & $-2,8 \%$ & 40.444 & 47.872 & $+18,4 \%$ \\
\hline \multicolumn{7}{|l|}{ Ambulatorial } \\
\hline Alta complexidade ambulatorial & 135.118 & 111.041 & $-17,8 \%$ & 159.667 & 139.692 & $-12,5 \%$ \\
\hline Atenção básica & 333.275 & 277.624 & $-16.7 \%$ & 77.720 & 0 & $-100 \%$ \\
\hline Ambulatorial estratégica & 18.659 & 16.862 & $-9,6 \%$ & 10.830 .199 & 26.522 .477 & $+144,9 \%$ \\
\hline \multirow[t]{2}{*}{ Média complexidade ambulatorial } & 1.315 .265 & 1.480 .711 & $+12,6 \%$ & 7.962 .380 & 9.809 .267 & $+23,2 \%$ \\
\hline & \multicolumn{3}{|c|}{ HE3 } & \multicolumn{3}{|c|}{ HE4 } \\
\hline Grupos de procedimentos & 2004 & 2007 & Variação & 2004 & 2007 & Variação \\
\hline \multicolumn{7}{|l|}{ Internação } \\
\hline Alta complexidade internação & 444 & 415 & $-6,5 \%$ & 2.518 & 2.384 & $-5,3 \%$ \\
\hline Internação estratégica & 52 & 21 & $-59,6 \%$ & 776 & 656 & $-15,5 \%$ \\
\hline Média complexidade internação & 8.659 & 9.859 & $+13,9 \%$ & 8.274 & 8.543 & $+3,3 \%$ \\
\hline \multicolumn{7}{|l|}{ Ambulatorial } \\
\hline Alta complexidade ambulatorial & 21.749 & 35.467 & $+63,1 \%$ & 70.178 & 105.222 & $+49,9 \%$ \\
\hline Atenção básica & 170.630 & 46.494 & $-72,8 \%$ & 126.317 & 117.086 & $-7,3 \%$ \\
\hline Ambulatorial estratégica & 4.540 & 4.925 & $+8,5$ & 14.485 & 21.070 & $+45,5 \%$ \\
\hline Média complexidade ambulatorial & 766.551 & 973.036 & $+26,9 \%$ & 1.087 .815 & 1.302 .553 & $+19,7 \%$ \\
\hline
\end{tabular}

* Os dados referem-se aos valores apresentados segundo o SIH e SIA do DATASUS/MS fornecidos pelo MS em abril de 2009.

Todas as condições presentes no HE1, HE3 e HE4, à exceção de poucas sobre as quais os dirigentes não souberam informar, foram implantadas independentemente da contratualização. $O$ dirigente do HE2 identificou a atualização do CNES, do SIA e do SIH como decorrente desse processo.

\section{Caracterização da contratualização}

Segundo cadastro do MS, o HE1 e HE4 foram contratualizados em final de 2004, o HE2 e o HE3 no início de 2005.

Os gestores da SMS1, da SMS3 e da SMS4 referiram elaborar periodicamente, o primeiro bienalmente e os outros anualmente, planos de saúde para seus municípios. A SMS3 informou elaborar com outros municípios adjacentes um plano intermunicipal a cada três anos, tendo em vista que o HE3 atende a população daqueles municípios. Foi referido que os planos da SMS1 e da SMS3 serviram de referência para a elaboração dos POAs dos hospitais. A SES2 prevê elaborar planos de saúde que servirão de base para o POA do hospital. Somente duas secretarias referiram elaborar contratos com os hospitais base- ados no conhecimento sobre as necessidades de atendimento de sua população e da rede de atenção, condição essencial para que o contratante saiba o que solicitar ao hospital contratado.

Os gestores da SMS1, da SES2 e SMS3 informaram a existência de Comissão de Acompanhamento dos Convênios/Contratos, com representantes da secretaria, hospital e usuários. A SMS4 não possui ainda Comissão (Tabela 4).

Os gestores da SMS1 e da SES2 informaram que as avaliações realizadas pelas Comissões ocorrem, respectivamente, a cada dois e três meses e que são formalizadas em relatório. O gestor da SMS3 informou não haver periodicidade pré-estabelecida e nem elaboração de relatório e o da SMS4 referiu que quando a Comissão for constituída se reunirá a cada três meses e formalizará a avaliação em relatório (Tabela 4).

O representante da SMS1 na Comissão monitora a produção do hospital mensalmente e, a cada dois meses, nos intervalos da reunião da Comissão, visita o hospital para verificar pendências identificadas nas avaliações anteriores, resultando em relatório que pontua para o pagamento da parte variável do convênio/contrato. 
Tabela 2a. Estruturas e práticas de qualidade assistencial e relação com a contratualização segundo os dirigentes dos Hospitais de Ensino - 2008.

\begin{tabular}{|c|c|c|c|c|}
\hline \multirow[b]{2}{*}{ Estruturas e práticas assistenciais } & \multicolumn{2}{|r|}{ HE1 } & \multicolumn{2}{|r|}{ HE2 } \\
\hline & Presença & $\begin{array}{c}\text { Relação } \\
\text { contratualização }\end{array}$ & Presença & $\begin{array}{c}\text { Relação } \\
\text { contratualização }\end{array}$ \\
\hline Patologia clínica $24 \mathrm{~h}$ & S & I & S & $\mathrm{I}$ \\
\hline Imagem $24 \mathrm{~h}$ & S & I & S & I \\
\hline Farmacêutico na Farmácia 24h & S & I & $S$ & I \\
\hline $\begin{array}{l}\text { Prontuário único (para pacientes externos e } \\
\text { internos) }\end{array}$ & S & I & S & I \\
\hline $\begin{array}{l}\text { Dispensação de medicamentos por dose unitária } \\
\text { (cada dose) ou por dose individualizada }(24 \mathrm{~h})\end{array}$ & S & I & $S$ & I \\
\hline Comissão de farmácia e terapêutica & S & I & S & I \\
\hline Comissão de infecção hospitalar com busca ativa & S & I & S & I \\
\hline Comissão de avaliação e revisão de prontuários & S & I & S & I \\
\hline Comissão de revisão de óbitos & S & $\mathrm{D}$ & S & I \\
\hline Comissão de ética médica & S & I & S & I \\
\hline Comissão de ética em pesquisa & $\mathrm{S}$ & I & S & I \\
\hline Comissão de mortalidade neonatal & $\mathrm{N}$ & NA & S & I \\
\hline Comissão de mortalidade materna & $\mathrm{S}$ & I & S & I \\
\hline Comissão de transplantes e captação de órgãos & S & I & S & I \\
\hline Comitê transfusional & S & I & $S$ & I \\
\hline Equipe multiprofissional de terapia nutricional & S & I & S & I \\
\hline Sessões clínicas periódicas & S & I & S & I \\
\hline \multirow[t]{2}{*}{ Diretrizes clínicas } & S & I & S & I \\
\hline & \multicolumn{2}{|r|}{ HE3 } & \multicolumn{2}{|r|}{ HE4 } \\
\hline Estruturas e práticas assistenciais & Presença & $\begin{array}{c}\text { Relação } \\
\text { contratualização }\end{array}$ & Presença & $\begin{array}{c}\text { Relação } \\
\text { contratualização }\end{array}$ \\
\hline Patologia clínica $24 \mathrm{~h}$ & S & I & S & I \\
\hline Imagem $24 \mathrm{~h}$ & S & I & S & I \\
\hline Farmacêutico na Farmácia 24h & S & I & S & I \\
\hline $\begin{array}{l}\text { Prontuário único (para pacientes externos e } \\
\text { internos) }\end{array}$ & S & I & S & I \\
\hline $\begin{array}{l}\text { Dispensação de medicamentos por dose unitária } \\
\text { (cada dose) ou por dose individualizada }(24 \mathrm{~h})\end{array}$ & S & I & S & I \\
\hline Comissão de farmácia e terapêutica & S & I & $S$ & I \\
\hline Comissão de infecção hospitalar com busca ativa & $\mathrm{S}$ & I & S & I \\
\hline Comissão de avaliação e revisão de prontuários & $\mathrm{N}$ & NA & S & I \\
\hline Comissão de revisão de óbitos & $\mathrm{S}$ & I & S & I \\
\hline Comissão de ética médica & $\mathrm{S}$ & I & $\mathrm{S}$ & I \\
\hline Comissão de ética em pesquisa & S & I & S & I \\
\hline Comissão de mortalidade neonatal & S & I & $\mathrm{N}$ & NA \\
\hline Comissão de mortalidade materna & $\mathrm{S}$ & I & $\mathrm{N}$ & NA \\
\hline Comissão de transplantes e captação de órgãos & $\mathrm{N}$ & NA & $\mathrm{S}$ & I \\
\hline Comitê transfusional & $\mathrm{S}$ & I & S & I \\
\hline Equipe multiprofissional de terapia nutricional & $\mathrm{S}$ & I & S & I \\
\hline Sessões clínicas periódicas & $\mathrm{S}$ & I & $S$ & I \\
\hline Diretrizes clínicas & S & I & S & $\mathrm{D}$ \\
\hline
\end{tabular}

$\mathrm{S}=\mathrm{Sim} ; \mathrm{N}=$ Não; $\mathrm{NA}=$ Não se aplica; $\mathrm{I}=$ Independente; $\mathrm{D}=$ Decorrente. 
Tabela 2b. Estruturas e práticas de qualidade assistencial e relação com a contratualização segundo os dirigentes dos Hospitais de Ensino - 2008

\begin{tabular}{|c|c|c|c|c|}
\hline \multirow[b]{2}{*}{ Estruturas e práticas assistenciais } & \multicolumn{2}{|r|}{ HE1 } & \multicolumn{2}{|r|}{ HE2 } \\
\hline & Presença & $\begin{array}{c}\text { Relação } \\
\text { contratualização }\end{array}$ & Presença & $\begin{array}{c}\text { Relação } \\
\text { contratualização }\end{array}$ \\
\hline Gestão de doença/patologia & $\mathrm{N}$ & NA & $\mathrm{N}$ & NA \\
\hline Gestão de caso/paciente & $\mathrm{N}$ & NA & $\mathrm{N}$ & NA \\
\hline Estudo variação da prática médica & $\mathrm{N}$ & $\mathrm{NA}$ & $\mathrm{N}$ & NA \\
\hline $\begin{array}{l}\text { Classificação das internações por gravidade e } \\
\text { utilização de recursos }\end{array}$ & $\mathrm{N}$ & NA & $\mathrm{S}$ & I \\
\hline Protocolos de enfermagem & $\mathrm{S}$ & I & S & I \\
\hline Ouvidoria & $\mathrm{S}$ & I & S & I \\
\hline Vigilância epidemiológica & $\mathrm{S}$ & I & S & I \\
\hline Hemovigilância & $S$ & I & S & I \\
\hline Farmacovigilância & $\mathrm{S}$ & I & S & I \\
\hline Tecnovigilância & $\mathrm{S}$ & I & S & I \\
\hline Vigilância da Saúde do trabalhador & $\mathrm{S}$ & I & S & I \\
\hline Plano de humanização formalmente implantado & $\mathrm{S}$ & I & S & I \\
\hline $\begin{array}{l}\text { Visitas médicas diárias na internação atualizando } \\
\text { prescrição e evolução }\end{array}$ & $\mathrm{S}$ & I & S & I \\
\hline $\begin{array}{l}\text { Visitas de enfermagem diárias na internação } \\
\text { atualizando prescrição e evolução }\end{array}$ & $\mathrm{S}$ & I & S & I \\
\hline Visitas diárias de familiars & $\mathrm{S}$ & I & S & I \\
\hline $\begin{array}{l}\text { Horários pré-estabelecidos para prestar } \\
\text { informações sobre o paciente pela equipe }\end{array}$ & $\mathrm{N}$ & NA & S & I \\
\hline $\begin{array}{l}\text { Avaliação de satisfação dos usuários ao menos } \\
\text { uma vez ao ano }\end{array}$ & $\mathrm{S}$ & I & S & I \\
\hline \multirow{2}{*}{ Balanço } & $29 / 35$ & - & $32 / 35$ & - \\
\hline & $82,9 \%$ & & $91,4 \%$ & \\
\hline & \multicolumn{2}{|r|}{ HE3 } & \multicolumn{2}{|r|}{ HE4 } \\
\hline Estruturas e práticas assistenciais & Presença & $\begin{array}{c}\text { Relação } \\
\text { contratualização }\end{array}$ & Presença & $\begin{array}{c}\text { Relação } \\
\text { contratualização }\end{array}$ \\
\hline Gestão de doença/patologia & $\mathrm{N}$ & NA & $\mathrm{N}$ & NA \\
\hline Gestão de caso/paciente & $\mathrm{N}$ & NA & $\mathrm{N}$ & NA \\
\hline Estudo variação da prática médica & $\mathrm{N}$ & $\mathrm{NA}$ & $\mathrm{N}$ & NA \\
\hline $\begin{array}{l}\text { Classificação das internações por gravidade e } \\
\text { utilização de recursos }\end{array}$ & $\mathrm{N}$ & NA & $\mathrm{N}$ & NA \\
\hline Protocolos de enfermagem & $\mathrm{S}$ & I & S & I \\
\hline Ouvidoria & $\mathrm{N}$ & NA & S & I \\
\hline Vigilância epidemiológica & $\mathrm{S}$ & I & NSI & NSI \\
\hline Hemovigilância & $\mathrm{S}$ & I & S & I \\
\hline Farmacovigilância & $\mathrm{S}$ & I & S & I \\
\hline Tecnovigilância & S & I & S & I \\
\hline Vigilância da Saúde do trabalhador & $\mathrm{N}$ & NA & S & I \\
\hline Plano de humanização formalmente implantado & S & $\mathrm{D}$ & NSI & NSI \\
\hline $\begin{array}{l}\text { Visitas médicas diárias na internação atualizando } \\
\text { prescrição e evolução }\end{array}$ & $\mathrm{S}$ & I & S & I \\
\hline $\begin{array}{l}\text { Visitas de enfermagem diárias na internação } \\
\text { atualizando prescrição e evolução }\end{array}$ & S & I & S & I \\
\hline Visitas diárias de familiars & S & I & S & $\mathrm{D}$ \\
\hline $\begin{array}{l}\text { Horários pré-estabelecidos para prestar } \\
\text { informações sobre o paciente pela equipe }\end{array}$ & NSI & NA & $S$ & $\mathrm{D}$ \\
\hline $\begin{array}{l}\text { Avaliação de satisfação dos usuários ao menos } \\
\text { uma vez ao ano }\end{array}$ & $\mathrm{N}$ & NA & $S$ & $\mathrm{D}$ \\
\hline \multirow[t]{2}{*}{ Balanço } & $25 / 35$ & - & $27 / 33$ & - \\
\hline & $71,4 \%$ & & $81.8 \%$ & \\
\hline
\end{tabular}

S = Sim; N = Não; NA = Não se aplica; NSI = Não sabe informar; I = Independente; D = Decorrente. 
Tabela 3. Estruturas e práticas de gestão e relação com a contratualização segundo os dirigentes dos Hospitais de Ensino $-2008$.

\begin{tabular}{|c|c|c|c|c|}
\hline \multirow[b]{2}{*}{ Estruturas e práticas de gestão } & \multicolumn{2}{|r|}{ HE1 } & \multicolumn{2}{|r|}{ HE2 } \\
\hline & Presença & $\begin{array}{c}\text { Relação } \\
\text { contratualização }\end{array}$ & Presença & $\begin{array}{c}\text { Relação } \\
\text { contratualização }\end{array}$ \\
\hline Conselho gestor (externo) & $\mathrm{N}$ & NA & $\mathrm{N}$ & NA \\
\hline Conselho diretor (interno) & $\mathrm{S}$ & I & $\mathrm{S}$ & I \\
\hline Outros colegiados & $\mathrm{S}$ & I & S & I \\
\hline Planejamento anual de atividades & $S$ & I & $\mathrm{S}$ & I \\
\hline Elaboração de plano diretor (documento) & $\mathrm{S}$ & I & S & I \\
\hline CNES atualizado & S & I & S & $\mathrm{D}$ \\
\hline SIA atualizado & S & I & $\mathrm{S}$ & $\mathrm{D}$ \\
\hline SIH atualizado & $\mathrm{S}$ & I & S & $\mathrm{D}$ \\
\hline Sistemas de informação informatizados & S & I & S & I \\
\hline Monitoramento de indicadores & $\mathrm{S}$ & I & S & I \\
\hline Elaboração de orçamento & $\mathrm{N}$ & NA & S & I \\
\hline Sistema de custos implantado & $\mathrm{S}$ & I & $\mathrm{N}$ & I \\
\hline SIAFI atualizado & $S$ & I & $\mathrm{S}$ & I \\
\hline Incentivos de $\mathrm{RH}$ & S & I & S & I \\
\hline $\begin{array}{l}\text { Práticas de controle de material de consumo } \\
\text { (curva } A B C \text { e padronização) }\end{array}$ & S & I & $S$ & I \\
\hline $\begin{array}{l}\text { Mais de } 80 \% \text { de equipamentos biomédicos } \\
\text { patrimoniados }\end{array}$ & S & I & S & I \\
\hline $\begin{array}{l}\text { Mais de } 60 \% \text { equipamentos biomédicos sob } \\
\text { manutenção preventiva }\end{array}$ & $\mathrm{N}$ & NA & S & I \\
\hline \multirow[t]{3}{*}{ Balanço } & $14 / 17$ & - & $15 / 17$ & - \\
\hline & $82,4 \%$ & & $88,2 \%$ & \\
\hline & \multicolumn{2}{|r|}{ HE3 } & \multicolumn{2}{|r|}{ HE4 } \\
\hline Estruturas e práticas de gestão & Presença & $\begin{array}{c}\text { Relação } \\
\text { contratualização }\end{array}$ & Presença & $\begin{array}{c}\text { Relação } \\
\text { contratualização }\end{array}$ \\
\hline Conselho gestor (externo) & $\mathrm{N}$ & NA & $\mathrm{S}$ & NSI \\
\hline Conselho diretor (interno) & $\mathrm{S}$ & I & $\mathrm{N}$ & NA \\
\hline Outros colegiados & $\mathrm{N}$ & NA & $\mathrm{N}$ & NA \\
\hline Planejamento anual de atividades & $\mathrm{N}$ & NA & $\mathrm{N}$ & NA \\
\hline Elaboração de plano diretor (documento) & $\mathrm{N}$ & NA & $\mathrm{N}$ & NA \\
\hline CNES atualizado & $\mathrm{S}$ & I & $\mathrm{S}$ & I \\
\hline SIA atualizado & S & I & S & I \\
\hline SIH atualizado & S & I & S & I \\
\hline Sistemas de informação informatizados & S & I & $S$ & I \\
\hline Monitoramento de indicadores & $\mathrm{S}$ & I & $\mathrm{S}$ & I \\
\hline Elaboração de orçamento & $\mathrm{N}$ & NA & $\mathrm{N}$ & NA \\
\hline Sistema de custos implantado & $\mathrm{N}$ & NA & $\mathrm{N}$ & NA \\
\hline SIAFI atualizado & $\mathrm{S}$ & I & $\mathrm{S}$ & I \\
\hline Incentivos de RH & S & NSI & S & NSI \\
\hline $\begin{array}{l}\text { Práticas de controle de material de consumo } \\
\text { (curva } A B C \text { e padronização) }\end{array}$ & S & I & S & I \\
\hline $\begin{array}{l}\text { Mais de } 80 \% \text { de equipamentos biomédicos } \\
\text { patrimoniados }\end{array}$ & S & I & S & I \\
\hline $\begin{array}{l}\text { Mais de } 60 \% \text { equipamentos biomédicos sob } \\
\text { manutenção preventiva }\end{array}$ & $\mathrm{N}$ & NA & $\mathrm{N}$ & NA \\
\hline \multirow[t]{2}{*}{ Balanço } & $10 / 17$ & - & $10 / 17$ & - \\
\hline & $58,8 \%$ & & $58,8 \%$ & \\
\hline
\end{tabular}

S = Sim; N = Não; NA = Não se aplica; NSI = Não sabe informar; I = Independente; $\mathrm{D}=$ Decorrente. 
Tabela 4. Caracterização do processo de contratualização segundo os gestores das Secretarias de Saúde 2008.

\begin{tabular}{|c|c|c|c|c|}
\hline Processo de contratualização & SMS1 & SES2 & SMS3 & SMS4 \\
\hline \multicolumn{5}{|l|}{ Plano municipal e ou estadual de saúde } \\
\hline Elaborado periodicamente & S & $\mathrm{N}$ & S & $S$ \\
\hline Usado como referência para o POA & $\mathrm{S}$ & NA & $\mathrm{S}$ & NSI \\
\hline \multicolumn{5}{|l|}{ Plano de saúde intermunicipal } \\
\hline Elaborado periodicamente & $\mathrm{N}$ & $\mathrm{N}$ & $\mathrm{S}$ & $\mathrm{N}$ \\
\hline Usado como referência para o POA & NA & NA & S & NA \\
\hline $\begin{array}{l}\text { Comissão de Acompanhamento dos Convênios/contratos/POA } \\
\text { formalmente constituída, por ato do secretário, com representantes das } \\
\text { partes contratantes e dos usuários identificados }\end{array}$ & S & S & S & $\mathrm{N}$ \\
\hline \multirow{2}{*}{\multicolumn{5}{|c|}{$\begin{array}{l}\text { Periodicidade de Avaliação da Comissão de Acompanhamento de } \\
\text { Convênios/contratos/POA }\end{array}$}} \\
\hline & & & & \\
\hline Bimensal & S & - & - & - \\
\hline Trimestral & - & $\mathrm{S}$ & - & - \\
\hline Sem periodicidade & - & - & $\mathrm{S}$ & - \\
\hline Formalização da avaliação em relatório & S & S & $\mathrm{N}$ & $\mathrm{N}$ \\
\hline Outras avaliações e prestação de contas do convênio/contrato/POA & S & S & S & $\mathrm{N}$ \\
\hline
\end{tabular}

$\mathrm{S}=\mathrm{Sim} ; \mathrm{N}=$ Não; NA = Não se aplica; NSI = Não sabe informar

Além disso, a SMS1, a cada seis meses, faz uma prestação de contas sobre os hospitais contratualizados, junto ao Conselho Municipal de Saúde e, a cada ano, encaminha relatório para o Tribunal de Contas do Estado e para a Comissão de Orçamento da Prefeitura (Tabela 4).

A SES2 possui uma assessoria de nível central especificamente constituída para acompanhar os hospitais de ensino e subsidiar a Comissão de Acompanhamento e hospitais. Também referiu realizar visitas ao hospital quando a contratualização tem início, quando há mudança de diretor no hospital ou quando se faz necessário (Tabela 4).

A SMS3, apesar do não funcionamento regular da Comissão de Acompanhamento, mensalmente realiza avaliações do HE3 através da coordenação hospitalar e ambulatorial, do planejamento e da auditoria da secretaria. A cada ano, o planejamento elabora um relatório sobre o convênio/contrato e o POA. O gestor da SMS4 não referiu avaliações (Tabela 4).

Apesar das secretarias, exceto a SMS4, terem comissões constituídas, a avaliação efetiva da realização das atividades do POA e dos recursos gastos através de reuniões regulares só acontece na SM1 e SES2 que também realizam atividades extras às reuniões regulares, com visitas ao hospital.

Todos os dirigentes de hospitais informaram estar com a certificação renovada ou em processo, mas somente o HE1 e HE2 referiram renova- ção de POA e de seu orçamento, formalizada em termo aditivo, após um ano do primeiro convênio/contrato. O HE4 estava em fase de negociação para renovação do convênio/contrato e POA e o HE3 ainda sem ter retomado a negociação com a SM3 (Tabela 5).

Todos os hospitais informaram que o POA foi elaborado conjuntamente com suas respectivas secretarias. No caso do HE4, esta informação se refere à renovação que estava em curso no momento da entrevista. Metas de assistência, de inserção na rede e de gestão estão presentes nos POA de todos os hospitais, enquanto que de ensino e de pesquisa somente nos HE1 e HE3 (Tabela 5).

Os dirigentes do HE3 e do HE4 informaram que o componente fixo dos recursos dos convênios/contratos corresponde a $90 \%$ e o componente variável, a $10 \%$ enquanto que o dirigente do HE2 referiu $85 \%$ e $15 \%$, e o do HE1, $75 \%$ e $25 \%$, respectivamente (Tabela 5).

$\mathrm{O}$ gestor da SMS1 referiu que, enquanto o componente variável correspondeu a $10 \%$, não havia reação por parte do hospital quando não era alcançada a pontuação mínima necessária para recebê-lo integralmente. Na medida em que esse percentual foi elevado, o hospital passou a buscar mais ativamente o alcance das metas e indicadores. Há uma previsão pactuada entre secretaria e hospital que esse percentual venha 
Tabela 5. Caracterização do processo de contratualização segundo os dirigentes dos Hospitais de Ensino 2008 .

\begin{tabular}{|c|c|c|c|c|}
\hline Processo de contratualização & HE1 & HE2 & HE3 & HE4 \\
\hline Hospital com certificação renovada ou em processo & S & S & S & S \\
\hline POA elaborado de forma conjunta pela Secretaria e Hospital & S & S & S & S \\
\hline $\begin{array}{l}\text { POA e orçamento renovados após } 1 \text { ano do primeiro convênio/contrato } \\
\text { com termo aditivo }\end{array}$ & S & S & $\mathrm{N}$ & $\mathrm{N}$ \\
\hline \multicolumn{5}{|l|}{ Dimensões contempladas no POA com metas e indicadores } \\
\hline Assistência & S & S & S & S \\
\hline Inserção na rede & S & S & S & S \\
\hline Gestão & S & S & S & S \\
\hline Ensino & S & $\mathrm{N}$ & S & $\mathrm{N}$ \\
\hline Pesquisa & S & $\mathrm{N}$ & S & $\mathrm{N}$ \\
\hline \multicolumn{5}{|l|}{ Composição percentual dos recursos do convênio/contrato (\%) } \\
\hline parte fixa & 75 & 85 & 90 & 90 \\
\hline parte variável & 25 & 15 & 10 & 10 \\
\hline \multicolumn{5}{|l|}{ Condições de repasse da parte fixa } \\
\hline parcelas mensais sem atraso & S & $S$ & $S$ & S \\
\hline independente de avaliação & S & S & S & S \\
\hline \multicolumn{5}{|l|}{ Condições de repasse da parte variável } \\
\hline parcelas mensais sem atraso & S & $S$ & S & S \\
\hline dependente de avaliação & S & S & $\mathrm{N}$ & $\mathrm{N}$ \\
\hline Não cumprimento de metas do POA & S & S & S & NA \\
\hline \multicolumn{5}{|l|}{ Consequências pelo não cumprimento } \\
\hline Não recebimento integral da parte variável & S & $\mathrm{N}$ & $\mathrm{N}$ & NA \\
\hline Não recebimento dos recursos da parte fixa & $\mathrm{N}$ & $\mathrm{N}$ & $\mathrm{N}$ & NA \\
\hline Advertência da secretaria & $\mathrm{N}$ & $\mathrm{N}$ & S & NA \\
\hline $\begin{array}{l}\text { Metas e indicadores do POA negociados internamente com serviços e } \\
\text { equipes }\end{array}$ & $\mathrm{S}$ & $\mathrm{N}$ & $\mathrm{N}$ & S \\
\hline \multicolumn{5}{|l|}{ Monitoramento interno do POA com } \\
\hline periodicidade definida & S & $S$ & $\mathrm{~N}$ & $\mathrm{~N}$ \\
\hline responsáveis identificados & S & S & $\mathrm{N}$ & $\mathrm{N}$ \\
\hline \multicolumn{5}{|l|}{ Monitoramento interno do orçamento com } \\
\hline periodicidade definida & S & $\mathrm{N}$ & $\mathrm{N}$ & $\mathrm{N}$ \\
\hline responsáveis identificados & S & $\mathrm{N}$ & $\mathrm{N}$ & $\mathrm{N}$ \\
\hline Contratos internos com os serviços com & S & $\mathrm{N}$ & $\mathrm{N}$ & $\mathrm{N}$ \\
\hline documento formal assinado entre as partes & S & - & - & - \\
\hline $\begin{array}{l}\text { serviços assistenciais, de apoio ao diagnóstico e tratamento, } \\
\text { administrativos e logísticos }\end{array}$ & S & - & - & - \\
\hline exigência de projetos de serviços & S & - & - & - \\
\hline metas e indicadores estabelecidos associados e ou decorrentes do POA & S & - & - & - \\
\hline monitoramento de metas e indicadores & S & - & - & - \\
\hline prêmios e punições em função do alcance das metas & S & - & - & - \\
\hline
\end{tabular}

$\mathrm{S}=\mathrm{Sim} ; \mathrm{N}=\mathrm{Não}$; NA = Não se aplica.

corresponder a 50\% dos recursos do convênio/ contrato conforme preconizado pelo Programa.

O repasse da parte fixa se dá sem atraso, em parcelas mensais e sem qualquer exigência na SES2, SMS3 e SMS4 (Tabela 5). A SMS1 é a única que condiciona o repasse do recurso fixo ao cumprimento de metas de produção física e outras.

O componente variável se dá sem atraso e é condicionado à avaliação somente no HE1 e HE2. Porém, desconto efetivo pelo não cumprimento integral das metas somente o HE1 sofreu. O HE2, porque sempre atinge a pontuação mínima da faixa de variação, não sofreu desconto (Tabela 5). Tal resultado evidencia que o condicionamento dos recursos ao alcance das metas, condição que caracteriza a relação contratual, só de fato aconteceu entre a SMS1 e HE1 e a SES2 e HE2.

Somente no HE1, as metas e os indicadores do POA foram negociados internamente com os serviços. No processo vigente de renovação do 
convênio/contrato/POA do HE4, os serviços do hospital têm discutido e participado de reuniões com a secretaria (Tabela 5).

O monitoramento interno do POA e o orçamento, com periodicidade e responsáveis identificados, apenas o HE1 informou realizar; o HE2 o referiu somente para o POA (Tabela 5).

Somente o HE1 informou contratos internos entre direção e chefias de unidades funcionais (agrupamento de serviços), formalizados em documento, envolvendo serviços assistenciais e administrativos. Há projetos de serviços com metas e indicadores associados ao POA do hospital, os quais são monitorados e estão articulados a um sistema de incentivos que premia as equipes que cumprirem com os compromissos pactuados (Tabela 5). À exceção de gratificações financeiras individuais, as premiações envolvem melhorias nos serviços, incluindo aquisição de equipamentos e financiamento para participar de congressos.

Tais resultados evidenciam que apenas no HE1, o contrato estabelecido com a secretaria se desdobra em contratos internos com metas e indicadores e incentivos presentes, o que é desejável para assegurar que as metas do POA sejam alcançadas.

\section{Aspectos facilitadores, dificuldades e benefícios associados à contratualização}

Como aspectos facilitadores, dirigentes de hospitais e gestores de secretarias identificaram a integração pré-existente hospital na rede de serviços, a participação das equipes do hospital no processo de negociação do convênio/contrato e o POA e a estruturação gerencial pré-existente da secretaria e hospital, destacando-se a continuidade administrativa da cúpula dirigente.

Como dificuldades são mencionadas, a insuficiente integração do hospital na rede de atenção, a ausência e/ou insuficiência de sistemas de informações, a não adesão às metas do POA por parte das equipes do hospital, a inadequação do sistema de incentivo para o hospital e sua inexistência para as equipes assistenciais. Os aspectos culturais dominantes relacionados à não prestação de contas e à falta de foco nos resultados são também citados como dificuldades. Por fim, são ainda considerados obstáculos, a insuficiente capacidade gerencial da secretaria e do hospital para lidar com as exigências da lógica contratual, em especial para elaborar, negociar, monitorar e avaliar o POA e para estimar os recursos financeiros para sua execução; tais dificuldades são ampla- mente ressaltadas na literatura internacional como fragilidades nas experiências existentes. Algumas dessas condições são também citadas nas experiências internacionais à contratualização $0^{5-7}$.

Os benefícios advindos referem-se à maior inserção do hospital na rede, ao fortalecimento da capacidade de gestão do hospital e de regulação das secretarias, à mudança na lógica de financiamento e de repasse de recursos, à melhoria de desempenho e ao incremento da prestação de contas.

\section{Discussão}

Há limitada evidência na literatura internacional sobre o impacto dos arranjos contratuais no desempenho dos prestadores, especialmente nos países em desenvolvimento. Nos países desenvolvidos resultados aparentemente favoráveis são referidos quanto à melhoria da qualidade da assistência e ao uso mais eficiente dos recursos ${ }^{4,5}$.

Igualmente, os resultados encontrados nesse estudo não são conclusivos e em parte podem ser explicados pelo curto espaço de tempo da experiência contratual com os hospitais de ensino.

Parece ter sido na produção de atividades que a contratualização mais promoveu a alteração desejada; em parte pode ser decorrente da melhoria no registro, também meta do Programa. A prestação de contas, se não foi incrementada, ao menos foi instituída onde não existia e onde já havia, foi reforçada ${ }^{6}$.

É frágil a associação, percebida pelos dirigentes hospitalares, entre a contratualização e as práticas e estruturas de qualificação assistencial e gerencial existentes, assim como com a inserção do hospital na rede. A certificação mais do que a contratualização parece ter induzido à implantação de algumas daquelas práticas e estruturas. A contratualização no máximo reforçou a inserção do hospital na rede e as práticas e estruturas assistenciais e gerenciais já existentes ${ }^{3}$, particularmente o planejamento e a avaliação que são condições necessárias à melhoria do desempenho.

Várias diretrizes de gestão e da assistência preconizadas no Programa e constantes do POA já eram cumpridas pelos hospitais por exigência anterior do MS, pela certificação ou ainda por iniciativa dos hospitais. Uma possibilidade é que as exigências do Programa contidas nos contratos/ convênios eram pouco desafiadoras, expressando apenas as práticas e estruturas já existentes.

Parece haver uma associação entre complexidade assistencial e estruturação assistencial e ge- 
rencial e os hospitais mais estruturados são também aqueles que apresentam processos de contratualização mais desenvolvidos. Porém, não foi possível afirmar que a contratualização mais estruturada tenha impactado na estruturação gerencial e assistencial dos hospitais, tendo em vista a frágil associação já mencionada entre a contratualização e as práticas e estruturas qualificadoras da gestão e da assistência.

Talvez seja possível supor que hospitais e secretarias mais estruturados tenham contribuído para uma contratualização mais estruturada, o que parece ter acontecido com a SMS/HE1 e a SES/HE2. O HE1 e HE2 são os hospitais mais desenvolvidos e assim como a SM1 e a SES2 são as secretarias mais estruturadas tendo em vista os mecanismos e as práticas de monitoramento por elas praticadas e informadas no estudo.

O desenvolvimento da capacidade de governo da secretaria, enquanto titular na relação com o hospital é de suma importância para o sucesso da relação contratual ${ }^{17-20}$. A SMS1 parece assumir mais robustamente o papel de titular, sabendo o que demandar, utilizando diversos mecanismos de monitoramento e instâncias de avaliação e de prestação de contas, aplicando e aprimorando os incentivos, conforme preconizado pela Teoria da Agência ${ }^{17-19}$. Sua maior fragilidade reside nos incentivos às equipes do hospital que, embora existentes, não parecem suficientes.

Naquela Teoria, as transações sociais são analisadas como relações titular ou principal/agente onde o titular para alcançar seus objetivos, depende que agentes realizem tarefas pré-especificadas em um contrato. O agente é contratado para atuar em defesa dos interesses do titular, porém ele, agente, tem também seus próprios interesses o que precisa ser considerado ${ }^{17-19}$.

A partir dos problemas identificados e dos ajustes sugeridos pelos entrevistados, dos resultados do estudo e da bibliografia sobre as experiências internacionais, destacam-se aspectos a merecerem atenção para o aprimoramento da contratualização dos hospitais de ensino.

\section{Desenvolvimento contínuo da capacidade gerencial de secretarias e hospitais}

É percebida importante debilidade gerencial. Será necessário reunir recursos e esforços, papel que poderia vir a ser desempenhado pelo MS, articulando uma rede de apoio com diferentes instituições, órgãos de ensino e pesquisa, de representação dos hospitais e de secretarias, e mesmo de entidades privadas. Hospitais e secreta- rias mais estruturadas poderiam apoiar secretarias e hospitais menos estruturados.

Embora se reconheça que a contratualização é uma relação onde as partes evoluem de forma associada, prioridade deve ser atribuída às secretarias, tendo em vista sua função de titular/contratante e gestora da rede. De forma mais imediata, assumir como áreas de desenvolvimento os problemas, as dificuldades e as sugestões apontadas nesse estudo.

É imprescindível a constituição de um núcleo burocrático capaz de planejar, monitorar, avaliar e regular, induzindo e redirecionando sempre que necessário, os rumos da contratualização $0^{21-23}$.

\section{Revisão do POA}

O conteúdo do POA deve contemplar outras dimensões do desempenho organizacional, como a econômico-financeira e em especial a da qualidade assistencial que está fragilmente contemplada.

$\mathrm{Na}$ dependência do grau de desenvolvimento assistencial e gerencial do hospital e da secretaria, podem ser introduzidas metas mais ousadas, visando à melhoria contínua da qualidade.

Embora não seja possível padronizar os indicadores a serem monitorados tendo em vista a diversidade das experiências, é recomendável que o conjunto de indicadores seja restrito, focando os aspectos problemáticos ${ }^{1}$. Muitos dados desinformam porque inviabilizam e retiram o foco da avaliação. Embora seja mais fácil e menos oneroso obter informação sobre os processos, mensurar os resultados é um determinante crítico para o uso adequado da lógica contratual.

Adicionalmente, os resultados da análise precisam ser discutidos entre as partes em contrato porque pode haver fatores contextuais que os expliquem, desconhecidos para quem recebe a informação $0^{24}$.

\section{Revisão da estimativa de financiamento}

Orçamentos subestimados constituíram-se em importantes dificuldades relatadas pelos dirigentes entrevistados e também na literatura internacional ${ }^{3}$. Para se obter estimativas mais realistas, os hospitais deveriam apurar os custos das atividades ponderados pela gravidade dos casos atendidos, o que por sua vez exige informação de qualidade nos prontuários sobre o diagnóstico e o consumo de recursos.

$\mathrm{Na}$ inexistência de informação sobre os custos reais das atividades seria necessário ajustar periodicamente o orçamento desde que justifica- 
do e avalizado pela comissão de acompanhamento. Embora previsto no convênio/contrato, não parece ser uma prática vigente.

\section{Revisão das práticas e mecanismos de monitoramento e de avaliação}

Há comissões bem estruturadas e operantes que poderiam dar suporte àquelas que ainda não funcionam adequadamente. Práticas utilizadas com bons resultados poderiam ser recomendadas tais como, a vinculação dos hospitais a técnicos da secretaria para monitorá-los de forma mais cotidiana, a inclusão na comissão de acompanhamento de representantes das secretarias de saúde de municípios adjacentes cuja população tem o hospital como referência de atendimento, etc.

É preciso manter a regularidade das avaliações e intensificar o controle social, que embora conste como condição formal do contrato através de um representante dos conselhos estadual e ou municipal de saúde, possivelmente na prática esse controle ainda é incipiente. O caso SMS1/ HE1 pode ser considerado mais próximo desta condição.

\section{Reestruturação dos sistemas de informação}

É necessário articular os sistemas já existentes com as informações exigidas pela contratualização subsidiando o monitoramento do POA e a estimativa de seu orçamento.

Importa acompanhar uma série histórica por hospital contratualizado sobre os procedimentos realizados, os recursos financeiros do contrato e de outras fontes e os indicadores de monitoramento.

Vários países com sistemas nacionais de saúde e práticas de contratualização possuem sistemas de informações articulando dimensões econômicas e assistenciais, com composição de recursos por atividade, despesas, diagnóstico principal, comorbidades e nível de gravidade.

\section{Revisão e ampliação dos sistemas de incentivos}

A estrutura de incentivos é incompleta, não há incentivos para a secretaria associados a alguma exigência ou compromisso - que também inexiste - nem para as equipes profissionais no hospital induzindo-as a alcançar o desempenho acordado no POA com a secretaria.

Conforme preconizado pela Teoria da Agência o sistema de incentivos visa a induzir o com- portamento do agente hospital e equipes assistenciais, simultaneamente propiciando o "constrangimento e o incentivo à participação”, mecanismos que associados tornam a adesão mais interessante do que a não adesão, permitindo ainda e principalmente, que os interesses dos profissionais sejam também atendidos ${ }^{17,18}$.

A exceção da SM1/HE1, o repasse da parte fixa e variável não está sendo necessariamente atrelado ao desempenho e, além disso, o percentual relativo à parte variável é baixo em relação ao total de recursos. Repasse de recursos sem associação ao desempenho gera pouco ou nenhum incentivo ao aumento da produtividade, à melhoria da qualidade ou a qualquer introdução de novas práticas, pois acaba por estimular a organização a funcionar como sempre funcionou ${ }^{4}$.

A não aplicação das recompensas e punições desvirtua a lógica contratual e enfraquece a prestação de contas sobre o desempenho. Este é um aspecto chave para a Teoria da Agência, pois o desempenho organizacional é dependente dos arranjos institucionais utilizados e dos constrangimentos utilizados ${ }^{17-20}$.

Outro aspecto a ser revisto refere-se às faixas de variação de desempenho que possibilita ao hospital o recurso integral mesmo sem alcançar $100 \%$ das metas estabelecidas, o que parece fazer com que o hospital não empreenda todos os esforços que poderia para alcançar a meta integral.

\section{Ampliação da participação e da pactuação com os profissionais dos hospitais}

O hospital desenvolve um trabalho complexo centralmente dependente dos profissionais, os quais possuem autonomia e considerável poder dentro da organização ${ }^{24}$. Sem a sua adesão, a melhoria do desempenho não será alcançada.

O planejamento em organizações profissionais só poderá funcionar se for um processo desencadeado junto ao centro operacional ${ }^{24}$. A direção do hospital não executa o POA acordado com a secretaria sozinho, portanto precisa desdobrá-lo em planos específicos para seus serviços, de forma pactuada com as equipes ${ }^{5,23,24}$.

\section{Disseminação dos contratos internos e externos}

Tais planos devem ser objeto de contratos internos entre a direção e equipes. Além disso, a lógica contratual deveria ser disseminada para toda a rede de atenção. Na lógica da Teoria da Agência a prestação de contas deve se espalhar 
por toda a organização, construindo uma cadeia de cobranças e de prestação de contas por todo o sistema de saúde ${ }^{17}$.

\section{Articulação entre planejamento regional e contratualização}

O contrato deve vincular a execução local ao planejamento regional promovendo uma "amarração" formal do que a secretaria precisa e do que o hospital e outras unidades componentes da rede podem e se comprometem a realizar ${ }^{3}$.

\section{Contratualização como política de Estado}

Ter se constituído como política de governo já foi um importante avanço, entretanto, a estabilidade e o aprimoramento da contratualização requer que o Estado claramente se posicione através de uma política nacional de contratualização, sustentada por uma estrutura legal articulada à política nacional de saúde ${ }^{23}$, assegurando inclusive o suporte financeiro à sua manutenção, expansão e aperfeiçoamento ${ }^{5,21}$. Diminuiria o risco, nos casos de mudança de governos, de descontinuidade da experiência contratual e dos ganhos já conquistados. O recém Decreto do governo federal ${ }^{9}$ regulamentando o uso do contrato no SUS pode caminhar nesse sentido.

A introdução de práticas de maior responsabilização, prestação de contas e de controle sobre os resultados do hospital não se dá de forma descolada do contexto geral de baixa prestação de contas na administração pública, pois é dependente do legado cultural e da dinâmica política do país. A qualidade das regras e das instituições que regem os arranjos contratuais importa nos resultados alcançados, mas não os explicam integralmente ${ }^{22}$.

O foco no desenvolvimento gerencial pode cultivar uma cultura de abertura ao novo e à aprendizagem contínua, condições propiciadoras de mudanças culturais para que novas formas organizacionais (arranjo contratual) sejam incorporadas e instituídas novas práticas de trabalho onde a prestação de contas sobre o desempenho tenha lugar central.

O uso de arranjos contratuais é um processo evolutivo. Há uma fase de "aprender fazendo"3 antes deles se constituírem em efetivo instrumento de regulação e gestão ${ }^{5}$. Sua utilização pode propiciar um maior controle sobre a gestão pública, reduzindo erros, possibilitando o ajuste de rumo e favorecendo a aprendizagem contínua da organização, o que é essencial para a modernização do Estado e da administração pública ${ }^{22}$.

Destaca-se, entretanto que a resolução dos problemas dos sistemas e serviços de saúde precisa ser considerada a partir de uma visão sistêmica e articulada com diferentes macro-políticas. Exige incrementar a resolutividade da atenção básica, a conformar redes de serviços integradas, valorizar os recursos humanos, dentre outras iniciativas. Exige também, uma pactuação entre o Executivo, o Legislativo e o Judiciário, visando ajustar o financiamento do sistema de saúde brasileiro, como também aprimorar e flexibilizar a administração pública, de forma a, ao menos, minimizar os atuais entraves hoje enfrentados.

\section{Colaboradores}

SML Lima e FJU Rivera participaram igualmente de todas as etapas de elaboração do artigo.

\section{Agradecimentos}

Especial agradecimento aos dirigentes dos hospitais, secretarias de saúde e do Ministério da Saúde que foram entrevistados fornecendo o conteúdo para a tese de doutorado e ao $\mathrm{CNPq}$ (Conselho Nacional de Desenvolvimento Científico e Tecnológico), financiador do estudo. 


\section{Referências}

1. Forgia GM, Couttolenc BF. Desempenho Hospitalar no Brasil. Em Busca da Excelência. São Paulo: The World Bank, Instituto Brasileiro para Estudo e desenvolvimento do Setor de Saúde (IBEDESS), Editora Singular; 2009.

2. Loevinsohn B. Performance-Based Contracting for Health Service in Developing Countries. A Toolkit. Health, Nutrition, and Population Series. Washington: The World Bank; 2008.

3. Figueras J, Robinson RJ, Jakubowski E, editors. Purchasing to improve health systems performance. Maidenhead: European Observatory on Health Systems and Policies Series, Open University Press; 2005.

4. Mills A, Broomberg J. Experiences of Contracting: an overview of the literature. Geneva: WHO; 1998. (Technical Paper, Macroeconomics, Health and Development Series, number 33).

5. World Health Organization (WHO). The International Journal of Public Health, Special theme: contracting and health services. Bulletin of the World Health Organization 2006; 84(11).

6. Ashton T, Cumming J, McLean J. Contracting for health services in a public health systems: the New Zealand experience. Health Policy 2004; 69(1):21-31.

7. Loevinsohn B, Harding A. Contracting for the Delivery of Community Health Services: A Review of Global Experience. HNP Discussion Paper. EUA: The World Bank; 2004.

8. Brasil. Poder Executivo. Medida provisória n 520, de 31/12/2010. Diário Oficial da União 2010; $31 \mathrm{dez}$.

9. Brasil. Poder Executivo. Decreto ${ }^{\circ} 7508$, de 28/06/ 2011. Regulamenta a Lei no 8.080, de 19 de setembro de 1990, para dispor sobre a organização do Sistema Único de Saúde - SUS, o planejamento da saúde, a assistência à saúde e a articulação interfederativa, e dá outras providências. Diário Oficial da União 2011; 29 jun.

10. Brasil. Ministério da Saúde. Ministério da Educação. Portaria Interministerial no 1006, de 27/05/2004. Diário Oficial da União 2004; 28 maio.

11. Brasil. Ministério da Saúde. Gabinete do Ministro. Portaria ${ }^{\circ} 1702$, de 17/08/2004. Cria o Programa de Reestruturação dos Hospitais de Ensino no âmbito do Sistema Único de Saúde - SUS, e dá outras providências. Diário Oficial da União 2004; 18 ago.

12. Brasil. Ministério da Saúde. Gabinete do Ministro. Portaria $\mathrm{n}^{\circ} 1721$, de 21/09/2005. Cria o Programa de Reestruturação e Contratualização dos Hospitais Filantrópicos no Sistema Único de Saúde - SUS. Diário Oficial da União 2005; 22 set.
13. Ministério da Saúde. Gabinete do Ministro. Portaria ${ }^{\circ} 1044$, de 01/06/2004 Diário Oficial da União 2010; 2 jun.

14. Brasil. Ministério da Saúde e Ministério da Educação. Portaria Interministerial nº 2400, de 02/10/2007. Estabelece os requisitos para certificação de unidades hospitalares como Hospitais de Ensino. Diário Oficial da União 2010; 3 out.

15. Brasil. Ministério da Saúde. Gabinete do Ministro. Portaria $n^{\circ}$ 2532/GM de 26/10/2004. Diário Oficial da União 2010; 27 out.

16. Lima SML, Barbosa PR, Portela MC, Ugá MAD, Vasconcellos, MM, Gershman. Caracterização gerencial dos hospitais filantrópicos no Brasil. Cad Saude Publica 2004; 20(5):1249-1261.

17. Przeworski A. Sobre o desenho do Estado: uma perspectiva agente $x$ principal. In: Bresser Pereira LC, Spink PK, organizadores. Reforma do Estado e administração pública gerencial. Rio de Janeiro: Editora FGV; 1998.

18. Araújo M, Sanchez OA. A Corrupção e os Controles Internos do Estado. Lua Nova 2005; 65:137-173.

19. Pereira C. Em Busca de um Novo Perfil Institucional de Estado: uma Revisão Crítica da Literatura Recente. Revista Brasileira de Informação Bibliográfica em Ciências Sociais 1997; 44.

20. Melo MA. Governance e reforma do Estado: o paradigma agente X principal. Revista do Serviço Público 1996; 120(1):67-82.

21. World Health Organization (WHO). Consultation on Strategic Contracting in Health Systems. Synthesis of the meeting. Geneva: WHO; 2008.

22. Sano H, Abrucio FL. Promessas e Resultados da Nova Gestão Pública no Brasil: o caso das Organizações Sociais de Saúde em São Paulo. RAE 2008: 48(3):64-80.

23. Perrot J, Roodenbeke E, éditeurs. La Contractualisation dans les Systèmes de Santé. Pour une utilization effiae et appropriée. Paris: Éditions Karthala; 2005.

24. Mintzberg H. Ascensão e Queda do Planejamento Estratégico. Tradução de Maria Adelaide Carpigiani. Porto Alegre: Ed Bookman; 2004.

Artigo apresentado em 15/05/2011

Aprovado em 07/07/2011

Versão final apresentada em 30/07/2011 